\title{
Map-guided surgery for atrial fibrillation
}

Takashi Nitta, MD

Hiroya Ohmori, MD

Shun-ichiro Sakamoto, MD

Yasuo Miyagi, MD

Shigeto Kanno, MD

Kazuo Shimizu, MD

From the Department of Cardiothoracic Surgery, Nippon Medical School, Tokyo, Japan.

Read at the Eighty-fourth Annual Meeting of The American Association for Thoracic Surgery, Toronto, Ontario, Canada, April 25-28, 2004.

Received for publication May 11, 2004; revisions received Sept 5, 2004; accepted for publication Sept 20, 2004.

Address for reprints: Takashi Nitta, MD, Cardiothoracic Surgery, Nippon Medical School, 1-1-5, Sendagi, Bunkyo-ku, Tokyo 113-8603, Japan (E-mail: nitta@nms.ac.jp).

J Thorac Cardiovasc Surg 2005;129:291-9

0022-5223/\$30.00

Copyright @ 2005 by The American Association for Thoracic Surgery

doi:10.1016/j.jtcvs.2004.09.012
Background: Although current surgical procedures result in a high success rate for atrial fibrillation, they are not guided by electrophysiologic findings in individual patients and thus might include unnecessary incisions in some patients or be inappropriate for other patients. We sought to determine whether intraoperative mapping is beneficial for the surgical treatment of atrial fibrillation.

Methods: A 256-channel 3-dimensional dynamic mapping system with custommade epicardial patch electrodes was used to examine the atrial activation during atrial fibrillation and to determine the optimal procedure in 37 patients with continuous and 9 patients with intermittent atrial fibrillation intraoperatively.

Results: Surgical intervention for atrial fibrillation was not indicated in 3 patients in whom the atrial electrograms had a low voltage over a broad area. Concurrent, multiple, and repetitive activations arising from the pulmonary veins or left atrial appendage were observed in all patients. A simple left atrial procedure consisting of pulmonary vein isolation and left atrial incisions without any right atrial incisions was performed in 8 patients in whom the right atrial activation was passive, and all (100\%) were cured of atrial fibrillation. The radial procedure was performed in the remaining 35 patients, and $31(89 \%)$ of the patients were cured of atrial fibrillation. In this subset of patients, 10 exhibited reentrant or focal activation in the posterior left atrium between the right and left pulmonary veins and required an additional linear ablation on the posterior left atrium. The total amount of postoperative bleeding after the simple left atrial procedure was significantly less than after the radial procedure $(378 \pm 135$ vs $711 \pm 364 \mathrm{~mL}$, $P=.03$ ). The right and left atrial transport functions were well preserved after both the radial and simple left atrial procedures.

Conclusion: Intraoperative mapping facilitates determining the optimal procedure for atrial fibrillation in each patient.

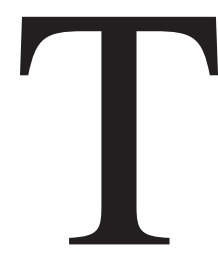

he traditional paradigm in surgical intervention for cardiac arrhythmias has been the electrophysiologic assessment of the arrhythmia, followed by the determination of a specific lesion for ablation or a definitive procedure on the basis of the results of the analysis in each patient. That has not been the case with the maze procedure for atrial fibrillation (AF). Development of the maze procedure was followed by extensive mapping studies in both animals and human subjects. ${ }^{1}$ However, the mapping data did not identify a stable and identifiable substrate suitable for ablation. The maze procedure consists of isolation of the posterior left atrium, including all 4 pulmonary veins, and multiple incisions on the right and left atria. ${ }^{2}$ The rationale behind the maze procedure is the confinement of the repetitive activation arising from the pulmonary veins to the isolated posterior left atrium and the interruption of all potential macroreentrant circuits by the atrial incisions. The maze procedure results in a high success rate for $\mathrm{AF}$ associated with or without structural heart 


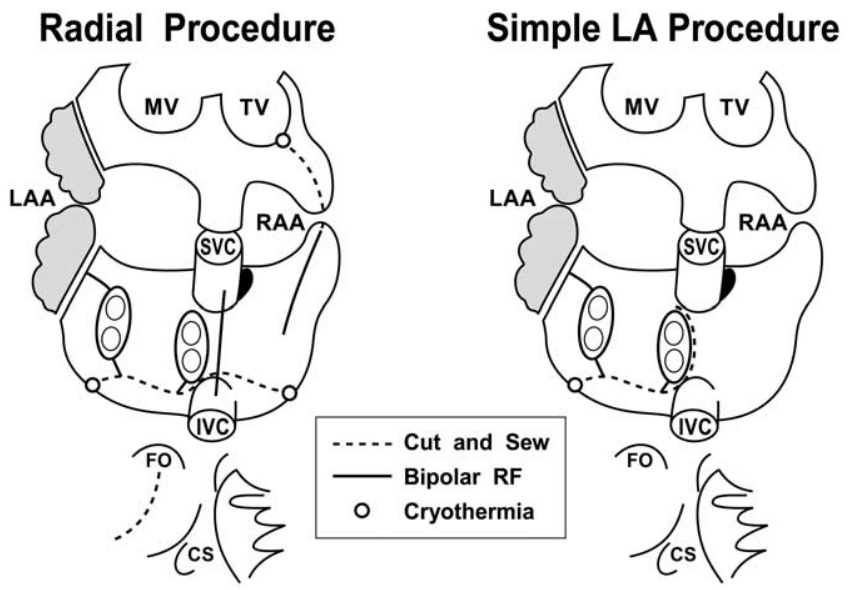

Figure 1. Schemas of the radial (left) and simple left atrial procedures (right). The upper, middle, and lower schemas represent the anterior and posterior views and the right-sided septal views of the atria, respectively. The 4 circles in the posterior left atrium indicate the pulmonary vein orifices. The broken lines indicate the cut-and-sew incisions, and the full lines indicate the bipolar radiofrequency linear ablation lines. The small circles indicate the cryothermia at the atrioventricular annuli. The radial procedure (left) is a modification of the maze procedure that preserves the posterior left atrium as a contractile portion with a physiologic activation and atrial transport function. The simple left atrial procedure (right) consists of bilateral pulmonary vein isolation, excision of the left atrial appendage, and a right-sided left atriotomy for the mitral valve procedure. There are no incisions in the right atrium or in the septum. $L A$, Left atrium; $M V$, mitral valve; $T V$, tricuspid valve; $R A A$, right atrial appendage; $L A A$, left atrial appendage; SVC, superior vena cava; IVC, inferior vena cava; FO, fossa ovalis; $C S$, coronary sinus; $R F$, radiofrequency.

disease. ${ }^{3}$ Because the procedure is not guided by electrophysiologic findings in individual patients, it might include unnecessary incisions in some patients or be inappropriate for other patients.

Recently, surgical procedures confined to the left atrium or the pulmonary veins have been shown to cure AF in selected patients. ${ }^{4,5}$ However, the success rate for AF with the limited procedures has been less than optimal, and the indications for these procedures have not been discussed in association with the electrophysiology of AF. We previously demonstrated, in an intraoperative mapping study of human AF, that multiple left atrial focal activations with fibrillatory conduction are the underlying mechanisms in permanent AF associated with mitral valve disease and that right atrial focal or reentrant activations are also observed in some patients. ${ }^{6}$ In the present study we sought to determine whether intraoperative mapping could characterize the electrophysiology of the AF and facilitate determining the optimal procedure in each patient.
TABLE 1. Pattern of atrial activation during atrial fibrillation

\begin{tabular}{llc}
\hline \multicolumn{1}{c}{ Left atrium } & \multicolumn{1}{c}{ Right atrium } & $\begin{array}{c}\text { No. of patients } \\
(\%)\end{array}$ \\
\hline Focal activation & Passive activation & $21(46)$ \\
Focal activation & Reentrant activation & $18(39)$ \\
Focal activation & Focal activation & $5(11)$ \\
Mitral reentrant activation & Passive activation & $1(2)$ \\
Passive activation & Reentrant activation & $1(2)$ \\
\hline
\end{tabular}

\section{Methods \\ Patients}

Intraoperative mapping was performed in 46 patients who underwent operations for AF. The study protocol was approved by the research committee of our institute, and written informed consent for an intraoperative electrophysiologic study was obtained from each patient. There were 23 male and 23 female patients with an average age of $63 \pm 10$ years. Thirty-eight $(83 \%)$ patients had valvular heart disease. There were 30 patients with mitral valve disease, 4 with aortic valve disease, and 4 with both aortic and mitral valve disease. Eleven of the patients with mitral valve disease had associated tricuspid valve regurgitation. Four patients with mitral valve disease also had coronary artery disease. Four patients had associated congenital heart disease, and 4 had no structural heart disease. There were 9 patients with intermittent AF and 37 with continuous AF. The duration of AF was 3 years or more in the patients with continuous AF. In the patients with intermittent $\mathrm{AF}$, the duration of $\mathrm{AF}$ ranged from 3 to 7 months. The average cardiothoracic ratio was $57 \% \pm 8 \%$. The left atrial dimension and left ventricular ejection fraction determined by means of transthoracic echocardiography were $50 \pm 11 \mathrm{~mm}$ and $60 \% \pm 14 \%$, respectively. The patients with a left atrial dimension of $70 \mathrm{~mm}$ or more were not indicated for AF surgery and thus were not subjected to this study.

\section{Intraoperative Mapping}

Intraoperative mapping was performed before the patient was cannulated for cardiopulmonary bypass. A 256-channel dynamic mapping system and custom-made mapping electrode patches were used as described previously. ${ }^{6}$ In brief, unipolar or bipolar electrograms were recorded from 253 atrial sites over the entire atrial epicardial surface, including the posterior left atrium between the right and left pulmonary veins. In the patients with intermittent AF, burst pacing at a pacing cycle length of $100 \mathrm{~ms}$ was applied on the right atrial appendage with a handheld electrode to induce AF. After the continuance of AF for more than 5 minutes was confirmed, the atrial electrograms were recorded. At least a 4000-ms duration of data was analyzed to characterize the activation pattern in each patient. Activation maps were displayed in a dynamic mode as a movie with 3-dimensionally constructed atrial models on a computer.

Reentry was defined as reactivation of a site by a wave front that continued from the preceding activation of the site. Focal activation was defined as an early activation without any late activation from a previous cycle adjacent to the earliest activation site. When an atrium was activated by a wave front that was 

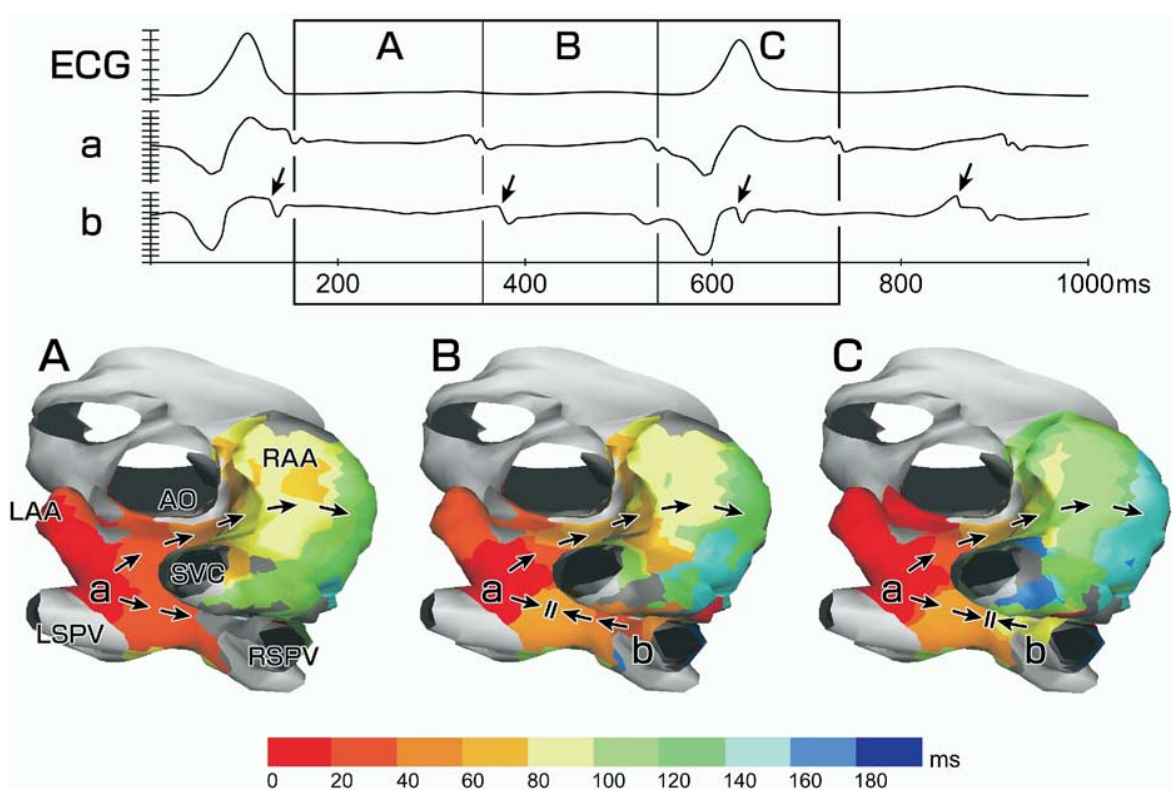

Figure 2. Atrial activation maps demonstrated in a 72-year-old male patient who had mitral valve regurgitation, coronary artery disease and intermittent AF. The maps represent the superior view of the atria. The atrial activation is exhibited with a color coding at $\mathbf{2 0}-\mathbf{m s}$ increments. The gray regions represent the regions in which no activation is seen during the time window. There were 2 foci of a repetitive activation: one in the left superior pulmonary vein (a) and the other in the right superior pulmonary vein (b). The activation maps during the time windows from $A$ to $C$ indicated in the electrocardiogram are shown. The duration of each time window (the cycle length of the activation recorded from electrode a) is 183, 203, and 203 ms, respectively. The arrows indicate the focal activation arising in the right superior pulmonary vein, and the cycle lengths of the activation are 244, 250, and $236 \mathrm{~ms}$. Because the activation from the left superior pulmonary vein was faster than that from the right superior pulmonary vein, it propagated toward the right atrium conducting over the Bachmann bundle. The right atrial activation was passive. $A O$, Aorta; $R A A$, right atrial appendage; $L A A$, left atrial appendage; SVC, superior vena cava; $L S P V$, left superior pulmonary vein; RSPV, right superior pulmonary vein.

traversed from the other atrium, the activation pattern was considered to be passive.

\section{Preference of Surgical Procedure for AF}

The pattern of atrial activation was analyzed by using the maps, and the definitive surgical procedure was determined for each patient. In the patients who exhibited reentrant or focal activation on the right atrium in addition to the focal activation in the left atrium, the radial procedure, ${ }^{7}$ a functional modification of the maze procedure, was performed. In the patients with right atrial passive activation that was conducted from the left atrial focal activation, a simple left atrial procedure was performed. The procedure consisted of bilateral pulmonary vein isolation, a right-sided left atriotomy to expose the mitral valve, and a left atrial appendage excision but no right atrial incisions. The atriotomy was extended down to the posterior mitral valve annulus between the middle and posterior scallops, and the annulus and coronary sinus were cryoablated. The incisions were connected to each other by means of linear cryoablation or a bipolar radiofrequency device (AtriCure Inc, West Chester, Ohio). The lesion set of each procedure is shown in Figure 1.

The right atrial incisions were made while the heart was beating, and the left atrial incisions were made after the heart was arrested. All the incisions were made by using a cut-and-sew technique, and cryothermia was used for the atrioventricular annuli, coronary sinus, and pulmonary vein isolation and its connecting lesions. In the latest 13 patients, a bipolar radiofrequency device was used for the pulmonary vein isolation and the other linear incisions on the right and left atria. Most patients had associated structural heart disease, and accordingly, concomitant procedures, such as mitral valve repair, were performed after the completion of the procedures for AF.

Because the right atrial incision was necessary in the patients with an atrial septal defect, all the patients underwent the radial procedure, even if the pattern of the right atrial activation was passive. All the right and left atrial incisions were made under an arrested heart condition in this subset of patients.

\section{Effect of the Simple Procedure}

The effect of the simple left atrial procedure guided by intraoperative mapping on the clinical results was evaluated from the cure rate of $\mathrm{AF}$, cardiopulmonary bypass time, aortic crossclamp time, total amount of postoperative bleeding, and postoperative atrial transport function. The postoperative follow-up periods ranged from 4 to 69 months, with an average of $38 \pm 23$ months. The 

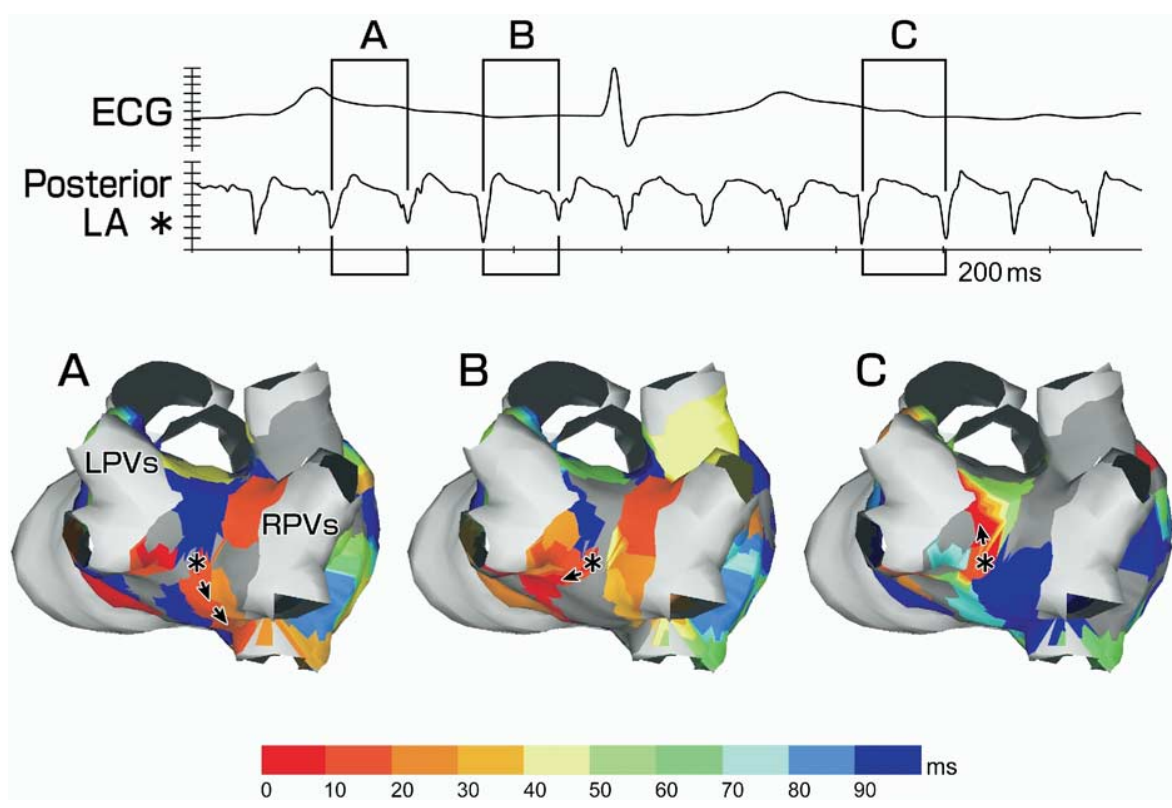

Figure 3. Nonpulmonary vein focus of a repetitive activation arising from the posterior left atrium between the right and left pulmonary veins. The maps were constructed in a 68-year-old male patient who had an atrial septal defect and continuous AF for 5 years. The electrogram shown along with the electrocardiogram was recorded at the focus $(*)$ in the posterior left atrium indicated in the maps. The maps represent the posterior view of the atria. The activation maps during the time windows $A, B$, and $C$ are shown. $L P V s$, Left pulmonary veins; $R P V s$, right pulmonary veins.

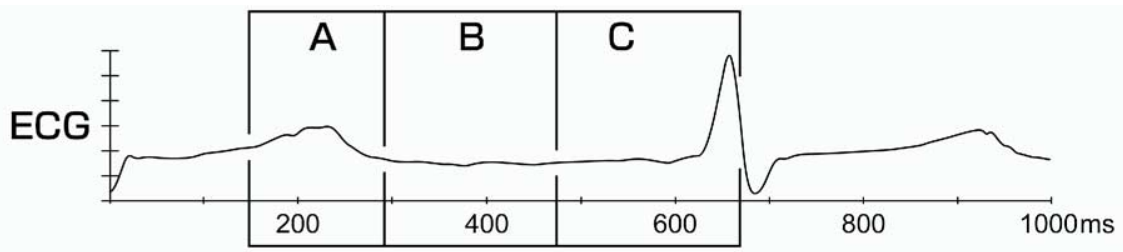

A

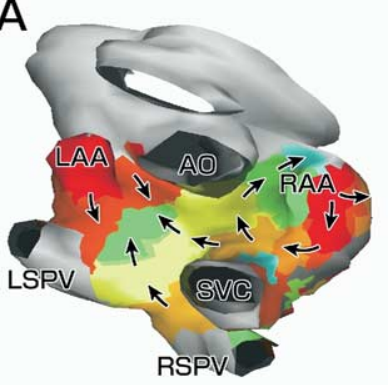

B

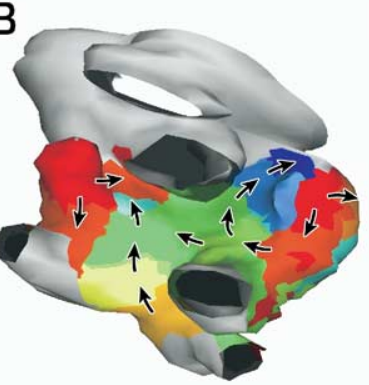

$\circ$

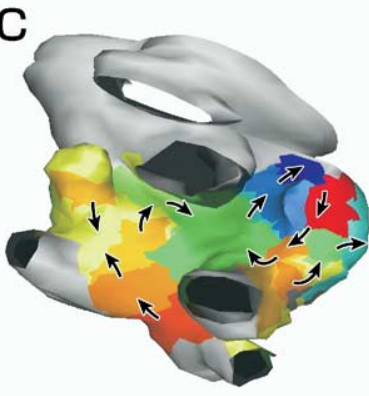

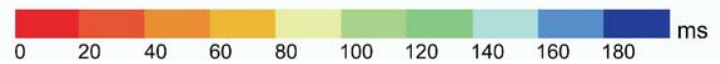

Figure 4. Reentrant activation in the right atrial appendage observed in a 68-year-old male patient who had mitral valve stenosis with regurgitation, tricuspid valve regurgitation, and continuous AF. In addition to the focal activations arising from the left atrial appendage and right superior pulmonary vein, there was a reentrant activation in the right atrial appendage. The reentrant activation collided with the focal activation originating from the left atrium. $A O$, Aorta; $R A A$, right atrial appendage; $L A A$, left atrial appendage; $S V C$, superior vena cava; $L S P V$, left superior pulmonary vein; $R S P V$, right superior pulmonary vein. 

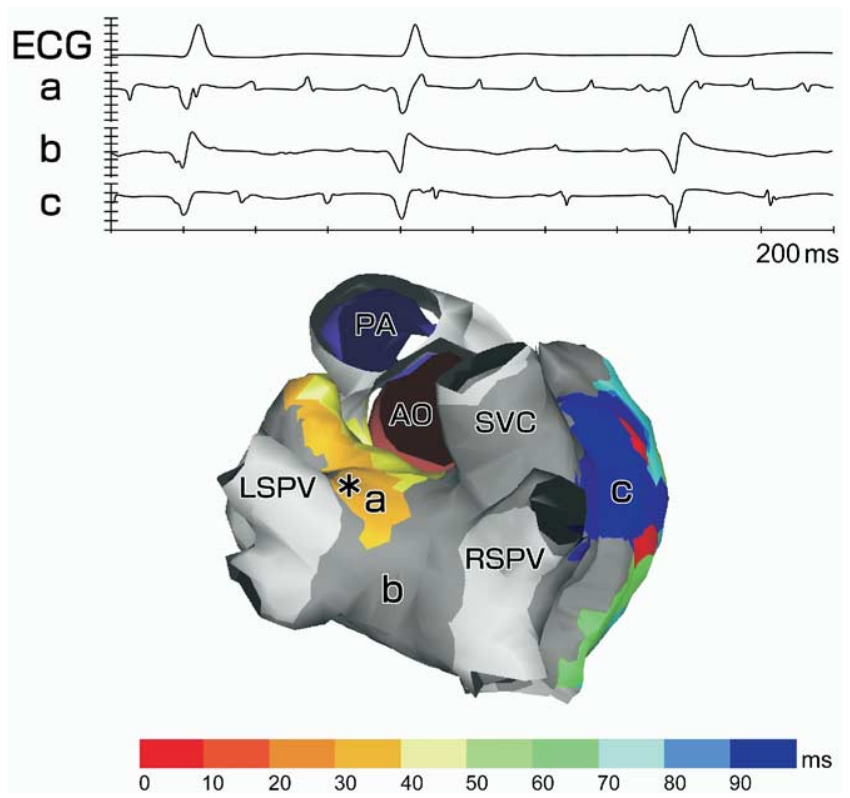

Figure 5. Low-voltage electrograms recorded over a broad area in the left atrium. The map was constructed in a 64-year-old female patient who had mitral valve stenosis with regurgitation and continuous AF. The map represents the right posterior view of the atria. The electrograms $a, b$, and $c$ were recorded from the left superior pulmonary vein, the posterior left atrium, and the lateral right atrium, respectively, as indicated in the maps. There was a rapid focal activation arising from the left superior pulmonary vein (*) and a passive activation in the right atrium. There was no electrical activity in the posterior left atrium. The gray color indicates the area with low-voltage electrograms. $P A$, Pulmonary artery; $A O$, aorta; $S V C$, superior vena cava; $L S P V$, left superior pulmonary vein; $R S P V$, right superior pulmonary vein.

postoperative cardiac rhythm was determined on the basis of the electrocardiogram recorded at the latest hospital visit. Because most patients had associated structural heart diseases and underwent concomitant procedures to correct any valvular or coronary artery disease, the cardiopulmonary bypass time and aortic crossclamp time for the simple left atrial procedure were compared with those of the radial patients matched for the concomitant procedures to justify the time required for the operation for $\mathrm{AF}$ and to eliminate the effect of the time required to perform the associated procedures. The total amount of postoperative bleeding collected through the chest tubes was measured. The atrial transport function was evaluated by means of transthoracic echocardiography 1 to 2 months postoperatively in all patients. The $\mathrm{A} / \mathrm{E}$ ratio, the ratio of the peak velocities of atrial filling (A) and early filling (E) waves, and the atrial filling fraction (AFF), derived as a percentage of the time-velocity integral of atrial filling in relation to the total diastolic time-velocity integral, were determined by means of planimetry of the diastolic mitral and tricuspid flow-velocity spectra, as described previously. ${ }^{7}$ Continuous values were expressed as means $\pm 1 \mathrm{SD}$. The Student $t$ test was used to compare data between 2 groups when appropriate. An analysis of variance with the Bonferroni correction was used to compare the effects of the proce-
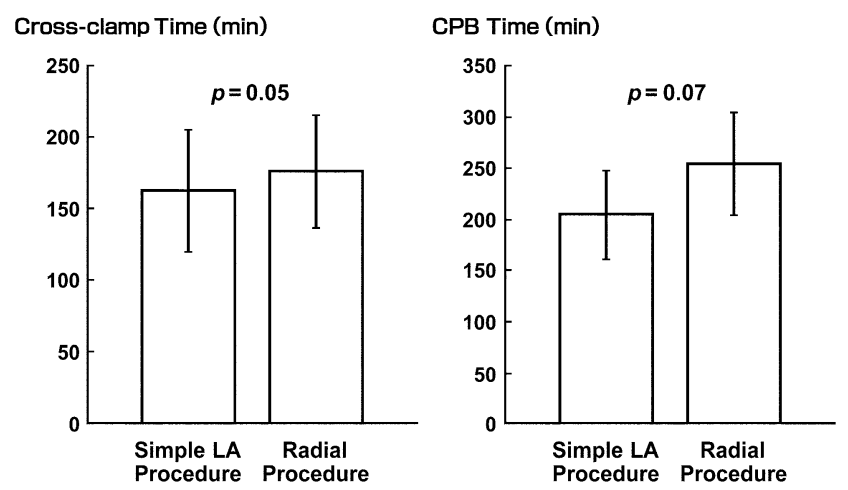

Figure 6. Crossclamp time (left) and CPB time (right) of the simple left atrial and radial procedures. CPB, Cardiopulmonary bypass; $L A$, left atrial.

dures and use of a bipolar radiofrequency device on the cardiopulmonary and aortic crossclamp times and the total amount of postoperative bleeding.

\section{Results}

\section{Time Required for Intraoperative Mapping}

All the patients with intermittent AF were inducible into sustained AF by burst pacing. The process for editing the local activation time and analyzing the activation maps was completed, usually within 15 minutes. However, it occasionally took 20 minutes or more, particularly in the patients with continuous AF. In 8 patients the atrial activation was extremely complex and could not be determined within 30 minutes. In the other patients, the atrial activation was successfully determined before starting bypass.

\section{Results of the Mapping}

The pattern of atrial activation is summarized in Table 1. Concurrent multiple repetitive activations arising from the pulmonary veins or the left atrial appendage were observed in most patients. Multiple focal activations with a different cycle length arose from multiple left atrial sites simultaneously. The fastest activation of the repetitive activations propagated toward the right atrium, leaving the other activations confined in a small atrial region (Figure 2). The number of left atrial foci ranged from 1 to 4 . The majority of the focal activations arose from the posterior left atrium adjacent to the right and left superior pulmonary veins or the left atrial appendage. In 10 patients the focal activations arose from the posterior left atrium between the right and left pulmonary veins (Figure 3 ). The activations originating from the posterior left atrium were usually confined to a small region because of the slower activation and longer pathway to the Bachmann bundle than the right or left superior pulmonary veins.

In $21(46 \%)$ patients the right atrial activation was passive, and no reentrant or focal activations were observed in 


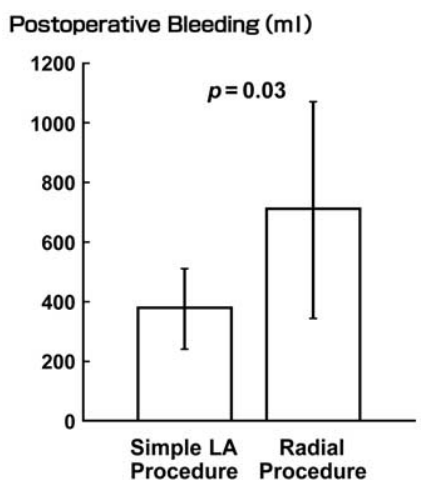

Figure 7. The total amount of postoperative bleeding after the simple left atrial and radial procedures. $L A$, Left atrial.

TABLE 2. The peak $A / E$ ratio and AFF across the mitral and tricuspid valves

\begin{tabular}{lcc}
\hline & $\begin{array}{c}\text { Simple left } \\
\text { atrial } \\
\text { procedure }\end{array}$ & Radial procedure \\
\hline $\begin{array}{l}\text { Transmitral Doppler flow } \\
\quad \text { Peak A/E ratio }\end{array}$ & $0.43 \pm 0.17$ & $0.41 \pm 0.16$ \\
$\quad$ AFF (\%) & $36.6 \pm 28.5$ & $25.4 \pm 16.0$ \\
$\begin{array}{l}\text { Transtricuspid Doppler flow } \\
\quad \text { Peak A/E }\end{array}$ & $0.65 \pm 0.29$ & $0.60 \pm 0.21$ \\
AFF (\%) & $33.1 \pm 7.0$ & $34.2 \pm 20.3$ \\
\hline
\end{tabular}

Data are presented as means $\pm 1 \mathrm{SD}$. The data were compared between the procedures by using the Student $t$ test. There was no statistically significant difference in any of the parameters. Peak $A / E$, Ratio of the peak velocity of the $A$ (atrial filling) wave to the $E$ (early filling) wave; $A F F$, atrial filling fraction.

the lateral right atrium. As the left atrial focal activation propagated toward the right atrium, there was a progressive conduction delay or block in the interatrial conduction pathways, such as the Bachmann bundle, resulting in an irregular and desynchronized right atrial activation. Progressive slowing of the conduction was also observed in the lateral right atrium. The delayed conduction resulted in residual activation from the preceding cycle and coexisting multiple wavelets in the lateral right atrium. As a result, the activation in the right atrium desynchronized with the left atrial activation and became irregular and complex.

Atrial reentry (Figure 4) and focal activations were observed in the lateral right atrium in 18 (39\%) and $5(11 \%)$ patients, respectively. The reentrant circuits changed their location and configuration cycle by cycle. The location of the focal activation was also variable and unstable.

There was one patient who exhibited a mitral macroreentrant activation with a passive activation in the right atrium. There was another patient who exhibited an unstable right atrial macroreentry with passive left atrial activation.

\section{Preference of the Procedures}

The preference of the surgical procedure was determined mainly by the pattern of the right atrial activation. In the patients who exhibited reentrant or focal activation on the right atrium, the radial procedure was performed. Of the 21 patients who exhibited passive activation in the right atrium, 8 patients underwent a simple left atrial procedure without any incisions in the right atrium. The other patients underwent the radial procedure because the pattern of atrial activation could not be completely determined in the intraoperative analysis. All 4 patients with atrial septal defects also underwent the radial procedure, even though 2 of them exhibited passive activation in the lateral right atrium. This is because an incision on the lateral right atrium was necessary to close the atrial septal defect.

In 3 patients the electrograms had a low voltage over a broad atrial area. The left atrial diameter was more than 65 $\mathrm{mm}$, and the cardiothoracic ratio was $65 \%$ or more in all these patients. Figure 5 shows the activation map observed in a patient. There was a focal activation arising from the left superior pulmonary vein and a passive activation in the right atrium. However, the electrograms in the broad area in the left atrium were low voltage, suggesting severely impaired electrical activity of the atrial myocardium. Operations for $\mathrm{AF}$ were not performed in these patients because of the low probability of converting the $\mathrm{AF}$ and the poor atrial contraction, even if sinus rhythm were to resume.

\section{Effects of the Simple Procedure}

There was one hospital death after the radial procedure not related to the intraoperative mapping or the operation for AF. There was no adverse effect of the intraoperative mapping procedure.

The cardiopulmonary bypass time for the simple left atrial procedure was $204 \pm 43$ minutes. Although this is shorter than the time required for the concomitant procedure-matched radial procedure (254 \pm 51 minutes), the difference did not reach statistical significance $(P=.07)$. The aortic crossclamp times for the simple left atrial procedure and the concomitant procedure-matched radial procedure were $162 \pm 43$ and $176 \pm 39$ minutes, respectively. There was no significant difference in the crossclamp time between the procedures $(P=.55$, Figure 6) The use of the bipolar radiofrequency device significantly shortened the cardiopulmonary bypass time $(P=.004)$ and insignificantly shortened the crossclamp time $(P=.06)$.

All the patients were cured of AF, and normal sinus rhythm resumed after the simple left atrial procedure. Thirty-one (89\%) of the 35 patients who underwent the radial procedure were cured of AF. There was no significant difference in the success rate for AF between the procedures.

The total amount of postoperative bleeding collected through the chest tubes after the simple left atrial procedure $(378 \pm 135 \mathrm{~mL})$ was significantly less than that collected 
after the radial procedure $(711 \pm 364 \mathrm{~mL}, P=.03$, Figure $7)$. The use of the bipolar radiofrequency device also decreased the postoperative bleeding $(P=.01)$.

The peak A/E ratio and AFF across the mitral and tricuspid valves after the simple left atrial procedure and the radial procedure are summarized in Table 2 . The right and left atrial transport functions were well preserved after both the radial and simple left atrial procedures. There was no significant difference in the peak $\mathrm{A} / \mathrm{E}$ ratio and AFF between the simple left atrial procedure and the radial procedure for across both the mitral and tricuspid valves.

\section{Discussion}

\section{Surgical Implications of Intraoperative Mapping}

The present study has demonstrated that the electrophysiology behind AF might be determined in each patient intraoperatively and that the AF might be cured by a more limited procedure. The intraoperative mapping successfully identified 8 patients who were perfectly indicated for a simple left atrial procedure that consisted of pulmonary vein isolation and left atrial incisions, and the success rate for curing AF was $100 \%$. Because the activation maps showed single or multiple left atrial focal activations and passive right atrial activation but no reentrant or focal activation in the right atrium, the right atrial incisions were not essential in terminating the $\mathrm{AF}$ in this subset of patients.

Pulmonary vein isolation alone has been shown to cure AF in selected patients. ${ }^{4,5}$ However, the success rate for curing AF has been described as approximately $70 \%$, which is significantly lower than that of the maze procedure. The patients who were not cured of AF by the pulmonary vein isolation might have reentry or focal activation in the right atrium and might be cured of AF by the right atrial incisions. Intraoperative mapping would be useful in determining the indications for simplified procedures for AF.

Three patients in whom the electrograms exhibited a low voltage over a broad atrial area did not undergo surgical intervention for $\mathrm{AF}$ in the present study. The left atrial diameter, cardiothoracic ratio, and duration of AF have been shown to predict the effect of the maze procedure on the postoperative cure of $\mathrm{AF}^{8}$ In fact, the left atrial diameter exceeded $60 \mathrm{~mm}$ in all 3 patients. However, there were other patients whose left atrial diameters were more than 60 $\mathrm{mm}$ but with atrial electrograms showing viable electrical activity. Those patients were cured of AF. Intraoperative mapping might be useful in predicting which patients will be cured of AF and in which patients a significant atrial contraction can be resumed postoperatively.

\section{Nonpulmonary Vein Foci}

Intraoperative mapping not only identifies the patients who will be cured of AF by means of a simple procedure, such as pulmonary vein isolation, but it might also suggest where surgeons should place an additional ablation to cure AF. An additional linear ablation was applied to the posterior left atrium between the right and left pulmonary veins in 10 patients who exhibited reentrant or focal activation in the posterior left atrium. Because we did not map the 2 patients who were not cured of AF postoperatively, even with the additional linear ablation in the posterior left atrium, the rationale for the recurrence of AF remained undetermined. A complete isolation of the entire posterior left atrium, including all 4 pulmonary vein orifices, as in the maze procedure, might have cured the AF in these patients.

Data from catheter mapping during pulmonary vein ablation have demonstrated that nonpulmonary vein foci can be seen in $14 \%$ to $18 \%$ of the patients with paroxysmal AF. ${ }^{9,10}$ The foci include the posterior left atrium, superior vena cava, crista terminalis, ligament of Marshall, coronary sinus ostium, and interatrial septum. A part of the failure or recurrence after the maze procedure might have been due to the nonpulmonary vein foci, and intraoperative mapping would be useful to identify those foci to eradicate the AF.

\section{Advantages of Limited Procedures}

Intraoperative mapping facilitates the indications for simple and limited procedures. ${ }^{11,12}$ If $\mathrm{AF}$ can be cured by using a simple and less-extensive procedure, ultimately, it is the patients who will benefit. The total amount of postoperative bleeding was significantly reduced, and the cardiopulmonary bypass time was shortened in the simple left atrial procedure used in this study. The lack of right atrial incisions and the shorter bypass time might have decreased the amount of postoperative bleeding. Because the lesion set on the left atrium in the simple left atrial procedure was the same as in the radial procedure, there was no difference in the left atrial transport function and aortic crossclamp time. If the intraoperative mapping is combined with minimally invasive ablation devices that enable off-pump surgery for $\mathrm{AF}$, it might further increase the number of patients who could benefit from the operation for AF.

Previous studies showed that there was a recurrence of AF or incidence of atrial flutter a few years after pulmonary vein isolation for $\mathrm{AF} .{ }^{5,13}$ Because the number of patients who underwent the limited procedure was small and the follow-up periods were relatively short in the present study, there is a chance that there might be the same outcome during a longer follow-up period, even after the map-guided limited procedure. Programmed electrical stimulation after completion of the procedure might be useful in determining the patients who should undergo additional right atrial incisions to prevent the recurrence of $\mathrm{AF}$ and atrial flutter.

\section{Limitations and Issues}

There are several issues that need to be solved before intraoperative mapping can be applied to the surgical treat- 
ment of $\mathrm{AF}$ as a routine procedure. First, the mapping technique needs to be improved, or a different modality that is capable of characterizing the atrial activation during $\mathrm{AF}$ within a minute needs to be developed. Even with highspeed CPU-based computerized mapping systems, activation time mapping is time consuming. This is because the atrial electrograms can be fragmented and have a low voltage in patients with $\mathrm{AF}$, and thus the analysis of the electrograms to determine the local activation time frequently requires extensive editing. Second, the interatrial septum and the coronary sinus should also be mapped because macroreentry conduction through the interatrial septum and interatrial electrical connections through the coronary sinus musculature or the septum can be mechanisms of AF. Preoperative endocardial catheter mapping would be useful in examining the endocardial activation. Finally, the long-term stability of the characteristics of the AF and the location of the sources of focal activation need to be confirmed.

\section{Conclusions}

Intraoperative mapping facilitates determining the optimal procedure for $\mathrm{AF}$ in each patient and might be useful in indicating which patients are suitable for AF operations and in elucidating nonpulmonary vein foci for additional ablation to eradicate the AF. The advantage of intraoperative mapping is most enhanced with the aid of off-pump ablative techniques. ${ }^{14,15}$ The results of the map-guided procedure should be confirmed with repeated mapping. The end point of the operation is the verification of conduction block across the incision, the disappearance of focal firing, and the inability to induce AF.

\section{References}

1. Cox JL, Canavan TE, Schuessler RB, Cain ME, Lindsay BD, Stone C, et al. The surgical treatment of atrial fibrillation. II. Intraoperative electrophysiologic mapping and description of the electrophysiologic basis of atrial flutter and atrial fibrillation. J Thorac Cardiovasc Surg. 1991;101:406-26.

2. Cox JL, Schuessler RB, D'Agostino HJ Jr, Stone CM, Chang BC, Cain ME, et al. The surgical treatment of atrial fibrillation. III. Development of a definitive surgical procedure. J Thorac Cardiovasc Surg. 1992; 104:1491-4.

3. Prasad SM, Maniar HS, Camillo CJ, Schuessler RB, Boineau JP, Sundt TM 3rd, et al. The Cox maze III procedure for atrial fibrillation: long-term efficacy in patients undergoing lone versus concomitant procedures. J Thorac Cardiovasc Surg. 2003;126:1822-8.

4. Sueda T, Nagata H, Shikata H, Orihashi K, Morita S, Sueshiro M, et al. Simple left atrial procedure for chronic atrial fibrillation associated with mitral valve disease. Ann Thorac Surg. 1996;62:1796-800.

5. Sueda T, Imai K, Ishii O, Orihashi K, Watari M, Okada K. Efficacy of pulmonary vein isolation for the elimination of chronic atrial fibrillation in cardiac valvular surgery. Ann Thorac Surg. 2001;71:1189-93.

6. Nitta T, Ishii Y, Miyagi Y, Ohmori H, Sakamoto S, Tanaka S. Concurrent multiple left atrial focal activations with fibrillatory conduction and right atrial focal or reentrant activation as the mechanism in atrial fibrillation. $J$ Thorac Cardiovasc Surg. 2004;127:770-8.

7. Nitta T, Lee R, Watanabe H, Harris KM, Erikson JM, Schuessler RB, et al. Radial approach: a new concept in surgical treatment for atrial fibrillation: II. Electrophysiological effects and atrial contribution to ventricular filling. Ann Thorac Surg. 1999;67:36-50.
8. Kawaguchi AT, Kosakai Y, Isobe F, Sasako Y, Eishi K, Nakano K, et al. Factors affecting rhythm after the maze procedure for atrial fibrillation. Circulation. 1996;94(suppl):II139-42.

9. Shah D, Haissaguerre M, Jais P, Hocini M. Nonpulmonary vein foci: do they exist? Pacing Clin Electrophysiol. 2003;26:1631-5.

10. Lin WS, Tai CT, Hsieh MH, Tsai CF, Lin YK, Tsao HM, et al. Catheter ablation of paroxysmal atrial fibrillation initiated by nonpulmonary vein ectopy. Circulation. 2003;107:3176-83.

11. Harada A, Konishi T, Fukata M, Higuchi K, Sugimoto T, Sasaki K. Intraoperative map guided operation for atrial fibrillation due to mitral valve disease. Ann Thorac Surg. 2000;69:446-50.

12. Yamauchi S, Ogasawara H, Saji Y, Bessho R, Miyagi Y, Fujii M. Efficacy of intraoperative mapping to optimize the surgical ablation of atrial fibrillation in cardiac surgery. Ann Thorac Surg. 2002;74:450-7.

13. Scharf C, Veerareddy S, Ozaydin M, Chugh A, Hall B, Cheung P, et al. Clinical significance of inducible atrial flutter during pulmonary vein isolation in patients with atrial fibrillation. $J$ Am Coll Cardiol. 2004;43:2057-62.

14. Maessen JG, Nijs JF, Smeets JL, Vainer J, Mochtar B. Beating-heart surgical treatment of atrial fibrillation with microwave ablation. Ann Thorac Surg. 2002;74:1307-11.

15. Prasad SM, Maniar HS, Schuessler RB, Damiano RJ Jr. Chronic transmural atrial ablation by using bipolar radiofrequency energy on the beating heart. J Thorac Cardiovasc Surg. 2002;124:708-13.

\section{Discussion}

Dr James L. Cox (Naples, Fla). I enjoyed Dr Nitta's presentation and congratulate him on some fine work, not only here today but also in the past. However, for clarification purposes, it is important for the audience to understand that once Dr Nitta added isolation of the pulmonary veins to the original radial procedure, it became identical to the final version of the maze procedure.

The concept of using electrophysiologic maps to guide AF surgery is mom and apple pie to arrhythmia surgeons because we have traditionally used maps to guide us in other types of arrhythmia surgery. With AF, however, things are a bit different. Cardiologists can now obtain these same beautiful maps by using only one intracavitary left atrial electrode rather than the extensive array of epicardial electrodes used by us 20 years ago and described by Dr Nitta today. Using a single electrode-tipped catheter, they are able to identify the triggers that reside in or around the pulmonary veins in many patients who have intermittent AF and then selectively ablate them. Because it now appears that encircling the pulmonary veins and blocking conduction across the left atrial isthmus might be the only requirements for curing most $\mathrm{AF}$, my first question for Dr Nitta is as follows: What is the practical reason for doing surgical epicardial mapping in the 21 st century? It would seem that you are suggesting that despite our ability to ablate virtually all AF with a very simple surgical procedure, we should still purchase this expensive mapping equipment. Do you believe that such an expense and extra operative time are really necessary, or would you recommend that intraoperative mapping be performed only by those surgeons who are specifically interested in learning more about the underlying electrophysiology of AF?

Dr Nitta. Thank you very much for your thoughtful comments. I believe that there are 2 advantages for using intraoperative mapping in AF operations. First, as I showed in the presentation, we can probably clearly identify those patients who are perfectly indicated for a simple procedure, such as just a pulmonary vein isolation procedure. In that subset of patients, we do not have to 
make a right atrial incision, and we can shorten the procedure and ischemic times.

The second advantage is that the success rate for AF operations can be improved by combining the intraoperative mapping with the off-pump ablation procedures. There are still recurrences of AF after the maze and radial procedures postoperatively. In those patients we might miss nonpulmonary vein foci or other unknown mechanisms that are not cured by the maze procedure. If we can combine intraoperative mapping with off-pump procedures, we can probably determine the mechanism of nonpulmonary vein foci, such as in the superior vena cava or posterior left atrium. Therefore there are indeed advantages for using intraoperative mapping in AF operations.

Dr Cox. I would like to ask one follow-up question in regard to the mapping procedure. I noticed that in your description of the patient with continuous AF, you mentioned "small multiple foci" that you believe to be driving the arrhythmia. I believe that terminology to be the crux of a lot of misunderstanding about the basic mechanism of AF. I would like to ask your opinion on this issue because there are 2 distinct theories as to the importance of those small microreentrant circuits.

Imagine a hurricane off the coast of Florida. It is well understood that hurricanes can spawn tornadoes, but it is obvious that tornadoes cannot generate hurricanes. Likewise, most electrophysiologists believe that large macroreentrant circuits definitely spin off small microreentrant circuits, but that it is extremely uncommon for small microreentrant circuits to generate and perpetuate large macroreentrant circuits. Indeed, if you believe that small microreentrant circuits are responsible for the larger macroreentrant circuits, then you have to explain how the radial procedure or the maze procedure can work because neither would be successful if this were the underlying mechanism for AF. Thus my final question is as follows: Do you believe that your studies indicate that continuous AF is maintained by these small microreentrant "rotors" in and around the pulmonary veins, or do you think it is maintained by the macroreentrant circuits themselves?
Dr Nitta. First of all, I need to say that I used the term "focal" to express the pattern of activation but did not mean automaticity. Furthermore, I believe the focal activation is probably microreentry in the pulmonary veins, and what we are looking at is just breakthrough of the microreentry in the pulmonary vein.

I do not believe that microreentry in the pulmonary veins makes a daughter reentry. What I have perceived from our mapping data is that microreentry in the pulmonary veins or other foci make repetitive activations, and the activation is so fast and cannot complete the activation of the entire atrium before the next repetitive activation arises. Therefore at the same time, there can be more than 2, sometimes 3, wave fronts originating from the same focus, that is, fibrillatory conduction. That is my understanding. If we isolate the foci, such as the pulmonary veins, the AF should be cured in most patients.

Dr Joao Q. Melo (Carnaxide, Portugal). Congratulations for a nice effort to better understand electrophysiology in AF. I am a bit surprised that you induce AF to do electrophysiologic assessment of AF even in patients who are in sinus rhythm. Our experience has been that to do a profound study of activation times, you need to have those patients in sinus rhythm. Therefore my question is as follows: Why are you inducing AF to do your electrophysiologic assessment of activation times? It is much easier, according to all the electrophysiologic studies, to do that in the sinus.

Dr Nitta. Of course, there is very important information in the mapping data during sinus rhythm, but I do not think we can localize the focal activation during sinus rhythm. We induced AF and usually waited for more than 5 minutes until it stabilized and then mapped the patient. We are still not sure that the mechanism of the induced $\mathrm{AF}$ is the same as in intermittent or spontaneous AF.

However, as I showed in the presentation, even in paroxysmal $\mathrm{AF}$ and induced $\mathrm{AF}$, the foci were in the pulmonary veins, which indicates that the mechanism of the induced AF is similar to that of the spontaneous AF. Therefore I believe that we should induce AF to find out the foci of repetitive activations. 